\title{
The prognostic value of cardiac SPECT performed at the primary care physician's office
}

\author{
Rami Doukky, MD, MSc, FACC, ${ }^{\mathrm{a}, \mathrm{b}}$ Nathan Frogge, MD, a Gautam Balakrishnan, \\ $M D,{ }^{a}$ Kathleen Hayes, MD, ${ }^{a}$ Fareed Moses Collado, MD, ${ }^{a}$ Maria O. Rangel, MD, a \\ Richard G. Trohman, MD, FACC, ${ }^{a}$ and Robert C. Hendel, MD, FACC, FAHA ${ }^{c}$
}

Background. The prognostic value of single-photon emission computed-tomography (SPECT)myocardial perfusion imaging (MPI) is well documented. However, the utility of SPECT-MPI when performed at a low-volume primary care physician's (PCP's) office is unknown.

Methods. We conducted a prospective cohort study of consecutive patients referred by their PCP to undergo a stress-MPI at the PCP's office using a mobile laboratory. Major adverse cardiovascular events (MACE) of death, myocardial infarction (MI), and coronary revascularization were prospectively tabulated using mail and telephone interviews, chart review, and social security death index.

Results. One thousand three hundred ninety subjects [mean age $58 \pm 13$ years; $44 \%$ women] were followed for $27 \pm 9$ months, with a 99\% complete follow-up rate. Subjects with abnormal MPI [174 (12.5\%)] had significantly higher rates of all-cause mortality [5.2\% vs $1.0 \%, P<.001]$, death, or MI $[5.7 \%$ vs $1.5 \%, P=.001]$, and the composite of death, MI, or late revascularization ( $>60$ days post-MPI) $[12.6$ vs $2.7 \%, P<.001]$. Overall MACE risk was associated with the total perfusion abnormality burden, while the revascularization rate was related to the reversible perfusion abnormality burden.

Conclusion. Contemporary SPECT-MPI performed in the setting of a PCP's office carries a robust prognostic value, similar to that reported in tertiary or large-volume practice settings. (J Nucl Cardiol 2013;20:519-28.)

Key Words: SPECT $\bullet$ myocardial perfusion imaging $(\mathrm{MPI}) \cdot$ prognosis $\bullet$ outcome $\bullet$ primary care physician (PCP)

\section{See related editorial, pp. 501-503}

From the Section of Cardiology, ${ }^{\text {a }}$ Rush University Medical Center, Chicago, IL; Division of Adult Cardiology, ${ }^{\mathrm{b}}$ John H. Stroger, Jr. Hospital of Cook County, Chicago, IL; and Division of Cardiology, ${ }^{\mathrm{c}}$ University of Miami Miller School of Medicine, Miami, FL.

Funding Investigator initiated research grant from Astellas Pharma US-Recipient: Rami Doukky. The funding source had no input in the study design, methods, data analysis, or reporting.

Received for publication Nov 16, 2012; final revision accepted Jan 11, 2013.

Reprint requests: Rami Doukky, MD, MSc, FACC, Section of Cardiology, Rush University Medical Center, 1653 W. Congress Pkwy, Chicago, IL 60612; rami_doukky@rush.edu.

$1071-3581 / \$ 34.00$

Copyright (c) 2013 American Society of Nuclear Cardiology.

doi:10.1007/s12350-013-9676-4

\section{INTRODUCTION}

Stress myocardial perfusion imaging (MPI) with single-photon emission computed-tomography (SPECT) has been extensively utilized in the last two decades to diagnose and risk-stratify patients with suspected or known coronary artery disease (CAD). ${ }^{1}$ This expansion in utilization was founded not only on favorable diagnostic performance, but also on robust long-term prognostic value. $^{2-5}$ Numerous studies have demonstrated that patients with normal MPI have an annual risk of major adverse cardiovascular events (MACE) of less than 1\%, while an abnormal MPI scan predicts a multifold increase in risk. ${ }^{2-6}$ Multiple studies demonstrated that the prognostic utility of MPI is incremental to clinical data, exercise treadmill test, and coronary angiography. ${ }^{7-9}$ In addition, MACE risk can be further defined by severity, size, and reversibility of perfusion abnormalities. ${ }^{2,9,10}$ However, previous MPI prognostic data were derived from tertiary care centers or a large-volume communitybased practice. ${ }^{2-5}$ More recently, the advent of mobile, dedicated cardiac SPECT imaging instruments allowed 
MPI to become an ambulatory commodity available to low-volume primary care practices. ${ }^{11}$ Although our group has shown that this practice is safe, ${ }^{11}$ the long-term prognostic value of MPI in a primary care physician's (PCP's) office has not been demonstrated.

The robust prognostic data of MPI may not necessarily be applicable to patients tested at the PCP's office. Such population is expected to have low prevalence and severity of CAD. ${ }^{12}$ Furthermore, a possible bias toward over-testing when point-of-care MPI is available may further decrease the likelihood of disease in this population, leading to deterioration of the positive predictive value of MPI. ${ }^{13}$ Thus, an MPI reported as "abnormal" may carry a lower MACE risk because of high falsepositive rates. Furthermore, in such a low-risk population, even a "truly" abnormal MPI may carry lower MACE risk than previously reported. In this investigation, we aimed to study the long-term prognostic value of stress-MPI performed at the PCP's office.

\section{DESIGN AND METHODS}

We conducted a 2-year follow-up, prospective cohortstudy of consecutive patients who were referred by their PCPs to undergo a clinically indicated rest/stress SPECT-MPI at the PCP's office in the period between August 15, 2007 and May 15,2010 . A total of 10 primary care practices encompassing 11 zip codes within the Chicago metropolitan area took part in the study. The practices varied in size from 1 to 6 practitioners, with a total of 20 PCPs (15 internists, 4 family practitioners, and 1 medicine-pediatrics physician). The volume of studies performed at each site varied from 2 to 32 MPIs per month. The technical staff and instrumentations were provided by a single accredited mobile nuclear cardiology laboratory. Exclusion criteria included the subject's failure to provide a valid address, telephone number, or social security number at the time of the MPI study or the PCP's refusal to provide the study personnel access to the patient's health records.

\section{Clinical Data}

Patients' baseline demographics, referral diagnosis, risk factors, cardiovascular history, and medications were tabulated at the time of the stress-MPI. If not otherwise specified, patients receiving anti-hypertensive medications were considered to be hypertensive, and those on lipid-lowering agents were considered to be dyslipidemic. Furthermore, we reviewed the last physician's note before MPI to determine the description of patient symptoms and the rationale for testing. If MPI was part of a pre-operative risk assessment, the type of operation was determined. The Framingham 10-year global CHD risk estimates were calculated. ${ }^{14}$ In addition, chest pain syndromes were classified into typical angina, atypical angina, and non-anginal chest pain on the basis of location, exacerbation with exercise, and resolution with rest. ${ }^{15}$ If the clinical notes did not describe the chest pain, then it was considered to be non-anginal. Dyspnea was considered an "angina equivalent" and classified as "non-anginal chest pain.", Consequently, we determined the pre-test probability of obstructive $\mathrm{CAD}$ based on age, gender, and chest pain classification using the Diamond and Forrester tables. ${ }^{15}$

A computer-based algorithm was applied to determine the level of adherence to the 2009 appropriate use criteria (AUC) in MPI referrals. ${ }^{16}$ Each study was assigned an appropriateness score (1 through 9) and was then classified as appropriate (score 7-9), uncertain (score 5-6), or inappropriate (score 1-4) according to the 2009 AUC. ${ }^{16}$

\section{Stress MPI}

A one-day rest/stress Technetium-99m sestamibi protocol, conforming to the American Society of Nuclear Cardiology guidelines, was implemented. ${ }^{17}$ As clinically appropriate, one of three stress modalities was used: the Bruce protocol exercise treadmill test, a standard 6-minute adenosine infusion, or adenosine-stress with low-level exercise (AdenoEX). ${ }^{18,19}$ Among those who underwent an exercise treadmill test, the Duke treadmill score (DTS) was calculated [exercise time (minutes) $-5 \times \mathrm{ST}$ segment deviation $(\mathrm{mm})-4 \times$ chest pain factor (0: none, 1: non-limiting angina, and 2: exercise limiting angina) $].^{20}$ All MPIs were acquired using a single mobile, upright acquisition, dual-head, dedicated cardiac SPECT camera (MAIcam $180^{\circledR}$, Mid Atlantic Imaging Services, Inc., Columbia, MD). No attenuation correction was applied.

MPI scans were processed and analyzed using QPS/QGS software (Cedars-Sinai Cardiac Suite, Los Angeles, CA). All MPIs were semiquantitatively interpreted by a single expert nuclear cardiologist (RD) who was blinded to the patients' exercise, clinical, and outcome data (August 2012). Using the 17 -segment model, the segmental radiotracer activity was scored according to the standard 5-point scale (0: normal; 1 : equivocal; 2: moderate; 3 : severe; and 4: absent). ${ }^{21}$ The semiquantitative summed stress (SSS), summed rest scores, and summed difference scores (SDS) were determined. The fully quantitative transient ischemic dilatation (TID) ratio and poststress gated-SPECT left ventricular ejection fraction (LVEF) were tabulated. An LVEF $<50 \%$ was considered abnormal. Myocardial perfusion was categorized as normal $(\mathrm{SSS}=0$ ) or abnormal (SSS $\geq 1$ ). Perfusion abnormalities were further classified based on their reversibility into fixed if SDS $=0$ or reversible if SDS $\geq 1$. An MPI scan was considered abnormal if either myocardial perfusion or post-stress gated-SPECT LVEF was abnormal.

\section{Outcomes}

Subjects were prospectively followed for events of death, myocardial infarction (MI), coronary artery bypass graft surgery (CABG), percutaneous coronary intervention (PCI), and coronary angiography. Four methods of ascertaining outcome events were uniformly applied in all patients: (1) review of patient health records (from July 2011 through February 2012) at the PCP's office; (2) two identical questionnaires mailed to patients' residences 6 months apart, in 
July 2011 and January 2012; (3) telephone interview questionnaires for subjects who did not complete the mailed surveys; and (4) a social security death index (SSDI) search performed in April 2012. The outcome assessors were blinded to MPI findings. Cause of death was determined from the death certificates retrieved from the Illinois Department of Public Health. MI events were defined based on the clinical determination of the treating cardiologist. Late coronary revascularizations, occurring $>60$ days post-MPI, were considered MACE as they were likely to be prompted by clinical deterioration or poor response to medical therapy. Early revascularizations ( $\leq 60$ days) were not considered MACE as they were likely to have been triggered-or biased-by the MPI findings. The primary endpoint was all-cause mortality. Secondary endpoints were (1) the composite endpoint of death or MI; and (2) the composite endpoint of death, MI, or late coronary revascularizations.

\section{Statistical Analysis}

We predicted an abnormal MPI rate of $11 \%$ based on published preliminary data. ${ }^{11}$ Thus, assuming a baseline annual mortality of $0.5 \%$ among subjects with normal MPI, we calculated that a cohort of 1,401 subjects would be needed to attain a $75 \%$ power to detect a four-fold increase in mortality rate associated with abnormal MPI after 2 years of follow-up (2-tailed $\alpha=0.05$ ). We targeted recruiting 1,500 subjects to allow some room for error in our assumptions.

We used the Fisher's exact test to compare dichotomous variables, which were each expressed as frequency (\%). The relative likelihoods of events were expressed as odds-ratios (OR) with 95\% confidence intervals (CI). The 2-tailed Student's $T$ test was used to compare continuous variables, which were expressed as mean \pm standard deviation (SD). Cox proportional-hazards models were used to compare event-free survival adjusted for covariates. Early revascularizations were considered '"non-events", and the affected subjects were followed in the model (not censored) until their first qualifying MACE or last clinical follow-up, whichever occurred first. Two-tailed $P$ values $\leq .05$ were considered significant. The SPSS version 18 software (SPSS, Inc., Chicago, IL) was used for all statistical analyses. The study was approved by the institutional review board of Rush University Medical Center.

\section{RESULTS}

We identified 1,546 consecutive subjects who were referred by their PCPs to undergo a rest/stress Tc-99m sestamibi SPECT-MPI study performed at the PCP's office. Among those, 142 subjects met one or more exclusion criteria: 68 did not have a valid SSN; 132 did not have a valid address or telephone number; and 43 due to their PCP's refusal to collaborate with the study. Of the remaining 1,404 eligible subjects, $14(1 \%)$ were lost to follow-up (10 had no return office visit and 4 had missing records), none of whom were identified as dead by SSDI. The remaining 1,390 subjects (99\%) had complete clinical follow-up.
Nineteen subjects had a repeat MPI, for whom outcome events were evaluated with reference to the first study. As compared with the study cohort with complete follow-up, the 156 patients who were excluded or lost to follow-up were younger (mean age $54 \pm 14$ vs $58 \pm 10$ years), trended to have lower likelihood of obstructive $\mathrm{CAD}$ $(18 \pm 13 \%$ vs $16 \pm 14 \%, P=.07)$ and had similar mean 10-year Framingham CHD risk $(11.9 \pm 10 \%$ vs $12.4 \pm 10 \%, P=.85)$ and nearly identical premorbid CAD prevalence (15\% in both groups, $P=.97$ ). Furthermore, other CAD risk factors, comorbidities, referral indication, and medication profile of the excluded group were not statistically different from the main study cohort.

The baseline characteristics for the study cohort are summarized in Table 1. Chest pain was the most

Table 1. Baseline characteristics

\begin{tabular}{ll}
\hline Total number of patients & 1,390 \\
Age, years (mean \pm SD) & $58 \pm 13$ \\
Women (\%) & $616(44)$ \\
MPI indication (\%) & \\
$\quad$ Chest pain syndrome & $653(47)$ \\
Dyspnea & $146(11)$ \\
Abnormal ECG-asymptomatic & $133(8)$ \\
Evaluation of known CAD- & $115(8)$ \\
$\quad$ asymptomatic & \\
Preoperative assessment & $30(2)$ \\
Asymptomatic/no symptoms & $248(18)$ \\
$\quad$ documented & \\
Framingham 10-year CHD Risk, & $12.5 \pm 10$ \\
$\quad \%$ (mean \pm SD) & $18 \pm 12$ \\
Likelihood of obstructive CAD*, & \\
$\quad \%$ (mean \pm SD) & $759(55)$ \\
Hypertension (\%) & $300(22)$ \\
Diabetes Mellitus (\%) & $613(44)$ \\
Hypercholesterolemia (\%) & $169(12)$ \\
Tobacco use (\%) & $505(36)$ \\
Family history of CAD (\%) & $29.7 \pm 5.7$ \\
BMI (kg/m ${ }^{2}$ ) & $202(15)$ \\
Known CAD (\%) & $46(3)$ \\
Prior CABG (\%) & $50(4)$ \\
Prior PCI (\%) & $24(1.7)$ \\
Prior MI (\%) & $297(21)$ \\
Antiplatelet (\%) & $498(36)$ \\
HMG-CoA reductase inhibitor (\%) & $250(18)$ \\
$\beta-B l o c k e r ~(\%)$ & $498(36)$ \\
ACE-I or ARB (\%) & \\
\hline
\end{tabular}

MPI, SPECT myocardial perfusion imaging; $C A D$, coronary artery disease; $C A B G$, coronary artery bypass grafting; $P C I$, percutaneous coronary intervention; $M I$, myocardial infarction; $A C E-I$, angiotensin converting enzyme inhibitor; $A R B$, angiotensin receptor blocker.

* Relevant to patients with chest pain or dyspnea. 
common symptomatic indication for stress MPI, followed by dyspnea. The likelihood of obstructive CAD among subjects with chest pain or dyspnea was $18 \pm 12 \%$. Based on the 2009 AUC, MPI referrals were appropriate in $709(51 \%)$, uncertain in $27(2 \%)$, and inappropriate in $654(47 \%)$. The inappropriate use rate varied widely between practitioners, ranging from $10 \%$ to $77 \%(P<.001)$. The stress modality used were exercise Bruce protocol in 1,101 (79\%) subjects, a standard 6-minute adenosine infusion in 138 (11\%) and AdenoEx in 131 (9\%). According to the DTS among those who underwent exercise stress, 869 (78.9\%) were stratified as low-risk and $231(21 \%)$ as intermediate-risk. Only one subject $(0.1 \%)$ was at high-risk and was analyzed within the intermediate-risk category.

MPI scans were normal in 1,216 (87.5\%) subjects. The remaining $174(12.5 \%)$ scans were abnormal: $115(66 \%)$ had abnormal perfusion, $28(16 \%)$ had LVEF $<50 \%$ but normal perfusion, and $31(18 \%)$ had abnormal perfusion and LVEF $<50 \%$. Among the 146 subjects with abnormal perfusion, $93(63.7 \%)$ had reversible perfusion abnormalities; $39(26.7 \%)$ had fixed perfusion abnormalities; and 14 $(9.6 \%)$ had fixed and reversible defects. Patients meeting the AUC had significantly higher rates of abnormal MPI scans [94 (13.3\%) vs 45 (6.9\%); OR 2.1 (1.4-3.0); $P<.001]$. Among the 156 patients who were excluded or lost to follow-up, 19 (12.2\%) had an abnormal MPI: 17 had abnormal perfusion (12 reversible, 4 fixed, 1 mixed); and 6 subjects had LVEF $<50 \%$ (4 of whom had abnormal perfusion and $\mathrm{LVEF}<50 \%$ ). The imaging characteristics in the excluded group were not statistically different from the 1,390 subjects with complete follow-up (all $P$ values $\geq .88$ )

Mean clinical follow-up time was $27 \pm 10$ months. The mean follow-up for mortality was $37 \pm 9$ months (clinical and SSDI data). During follow-up, there were $21(1.5 \%)$ deaths, $8(0.6 \%)$ nonfatal MIs, and $6(0.9 \%)$ cardiac deaths. In addition, $89(6.4 \%)$ subjects received coronary angiography, $45(3.2 \%)$ had PCI, and 11 (0.8\%) underwent CABG.

Using a Cox regression model, we identified that only two independent baseline clinical predictors of MACE were the 10-year Framingham CHD risk [HR 1.03 per $1 \%$ risk increment (CI 1.01-1.05), $P=.004]$ and premorbid CAD [OR 1.9 (CI 1.02-3.6), $P=.04$ ], while use of statin, aspirin, $\beta$-blockers, angiotensinconverting enzyme inhibitors, and angiotensin receptor blockers were not.

Patients with abnormal MPI had a higher rate of allcause mortality than those with a normal scan $[5.2 \%$ vs 1.0\%; OR 5.5 (CI 2.3-13.2); $P<.001$ ]. Furthermore, abnormal MPI was associated with higher rates of the composite of death or MI, cardiac death, and the composite of cardiac death or MI (Table 2). Even after

Table 2. Unadjusted risk of adverse cardiac events

\begin{tabular}{lcccc}
\hline & $\begin{array}{c}\text { Abnormal MPI } \\
(\mathbf{N}=\mathbf{1 7 4}) \mathbf{( \% )}\end{array}$ & $\begin{array}{c}\text { Normal MPI } \\
(\mathbf{N = 1 , 2 1 6 )}(\%)\end{array}$ & OR (95\% CI) & P value \\
\hline All-cause mortality & $9(5.2)$ & $12(1.0)$ & $5.5(2.3-13.2)$ & $<.001$ \\
Cardiac death & $4(2.3)$ & $2(0.2)$ & $14.3(2.6-78.6)$ & .003 \\
Nonfatal MI & $2(1.1)$ & $6(0.5)$ & $2.3(0.5-11.7)$ & .26 \\
Cardiac death or MI & $5(2.9)$ & $8(0.7)$ & $4.5(1.4-13.8)$ & .016 \\
Death or MI & $10(5.7)$ & $18(1.5)$ & $4.1(1.8-8.9)$ & .001 \\
Death, MI, or late & $22(12.6)$ & $33(2.7)$ & $5.2(2.9-9.1)$ & $<.001$ \\
$\quad$ revascularization & & & & \\
Angiography & $53(30.5)$ & $36(3.0)$ & $14.4(9.0-22.8)$ & $<.001$ \\
PCI & $27(16.0)$ & $18(1.5)$ & $12.2(6.6-22.7)$ & $<.001$ \\
$\quad$ Early PCI & $18(10.3)$ & $2(0.2)$ & $70.0(16.1-304.7)$ & $<.001$ \\
Late PCI & $9(5.2)$ & $16(1.3)$ & $4.1(1.8-9.4)$ & .002 \\
CABG & $8(4.6)$ & $3(0.2)$ & $19.5(5.1-74.2)$ & $<.001$ \\
$\quad$ Early CABG & $5(2.9)$ & $0(0)$ & NA* & \\
Late CABG & $3(1.7)$ & $3(0.2)$ & $7.1(1.4-35.4)$ & .0029 \\
Revascularization (CABG & $30(17.2)$ & $21(1.7)$ & $11.9(6.6-21.3)$ & $<.001$ \\
$\quad$ or PCI) & & & & \\
$\quad$ Early revascularization & $19(10.9)$ & $2(0.2)$ & $74.4(17.2-322.5)$ & $<.001$ \\
Late revascularization & $12(6.9)$ & $19(1.6)$ & $4.7(2.2-9.8)$ & $<.001$ \\
\hline
\end{tabular}

* OR cannot be calculated (zero events in the normal MPI group).

$¥$ Late revascularizations: $>60$ days post MPI; early revascularization: $\leq 60$ days post MPI. 

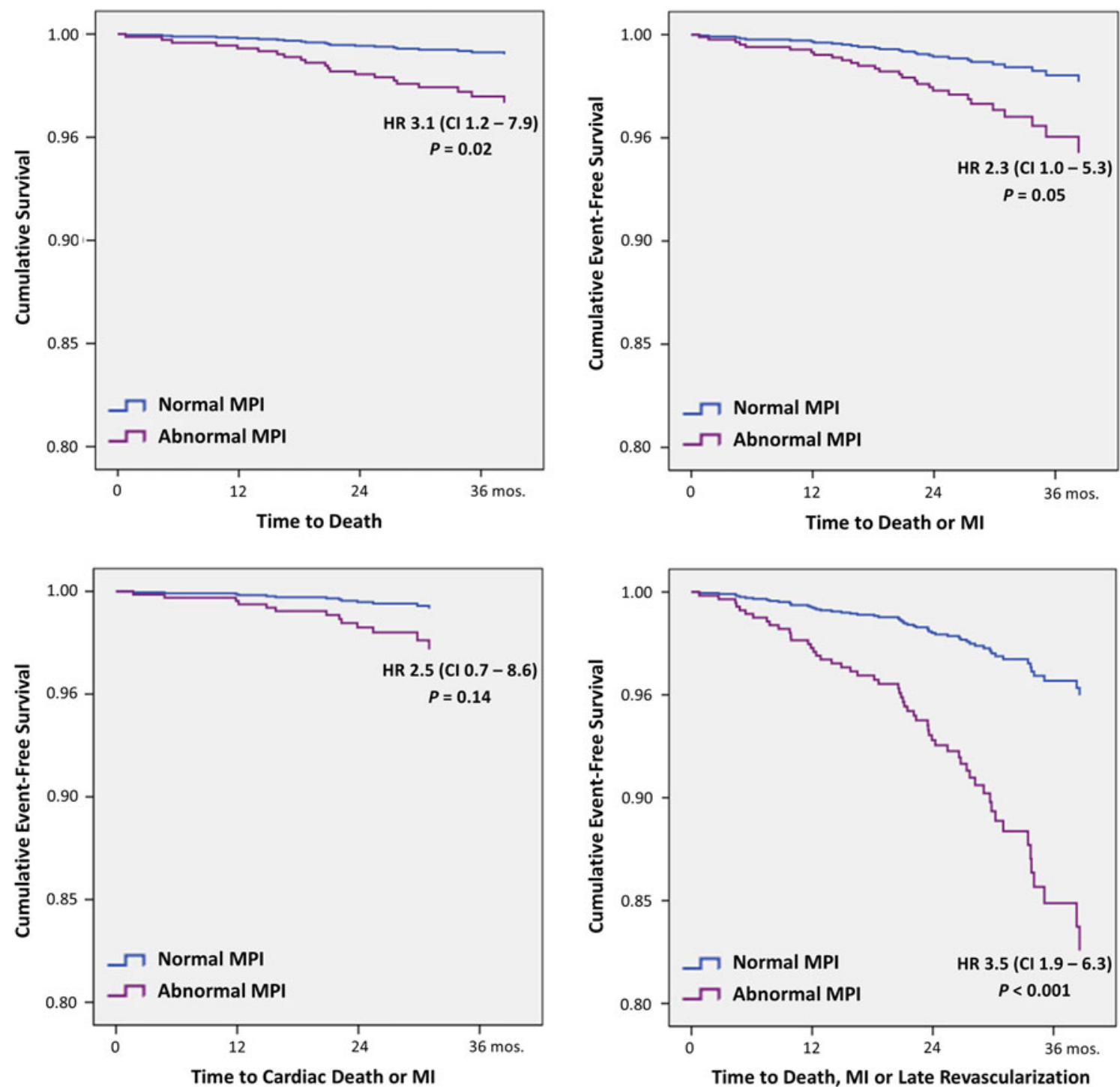

Figure 1. Cox proportional hazards survival curves of major study endpoints. Survival curves depict event-free survival of patients with normal vs abnormal MPI, adjusted for CAD status and 10-year Framingham CHD risk.

adjusting for Framingham CHD risk and CAD status, an abnormal MPI was an independent predictor for allcause mortality [HR 3.1 (CI 1.2-7.9); $P=.02$ ] and the composite of death or MI [HR 2.3 (CI 1.0-5.3); $P=.05$ ] (Figure 1). Patients with abnormal MPI tended to have a higher rate of nonfatal-MI, but the difference was not statistically significant $[1.1 \%$ vs $0.5 \% ; \quad P=.26]$. Patients with normal MPI had very low annualized rates of death $(0.32 \%)$, death or MI $(0.54 \%)$, and cardiac death or MI $(0.29 \%)$.

Patients with abnormal MPI had a higher mean TID ratio $(1.0 \pm 0.20$ vs $0.94 \pm 0.16, P<.001)$ and lower mean LVEF [52 $\pm 13 \%$ vs $64 \pm 8 \%, P<.001]$. Furthermore, both TID and LVEF were found to be incrementally predictive of all-cause mortality beyond Framingham CHD risk, CAD status, and myocardial perfusion [TID: HR 1.2 per 0.1 point increment (CI 1.01-1.3), $P=.03$; LVEF: HR 2.1 per 10 point decrement (CI 1.4-3.1), $P<.001]$. Depressed post-stress LVEF $(<50 \%)$ had a very strong and incremental prognostic value in predicting all-cause mortality in patients with and without perfusion abnormalities, even after adjusting for Framingham CHD risk and CAD status (Figure 2). Furthermore, TID $\geq 1.2$ demonstrated an incremental prognostic value in predicting allcause mortality beyond perfusion abnormality and depressed LVEF [HR 3.3 (CI 1.0-11.4), $P=.05$ ].

Patients with abnormal MPI had a significantly higher rate of coronary angiography [30.5\% vs $3.0 \%$ 

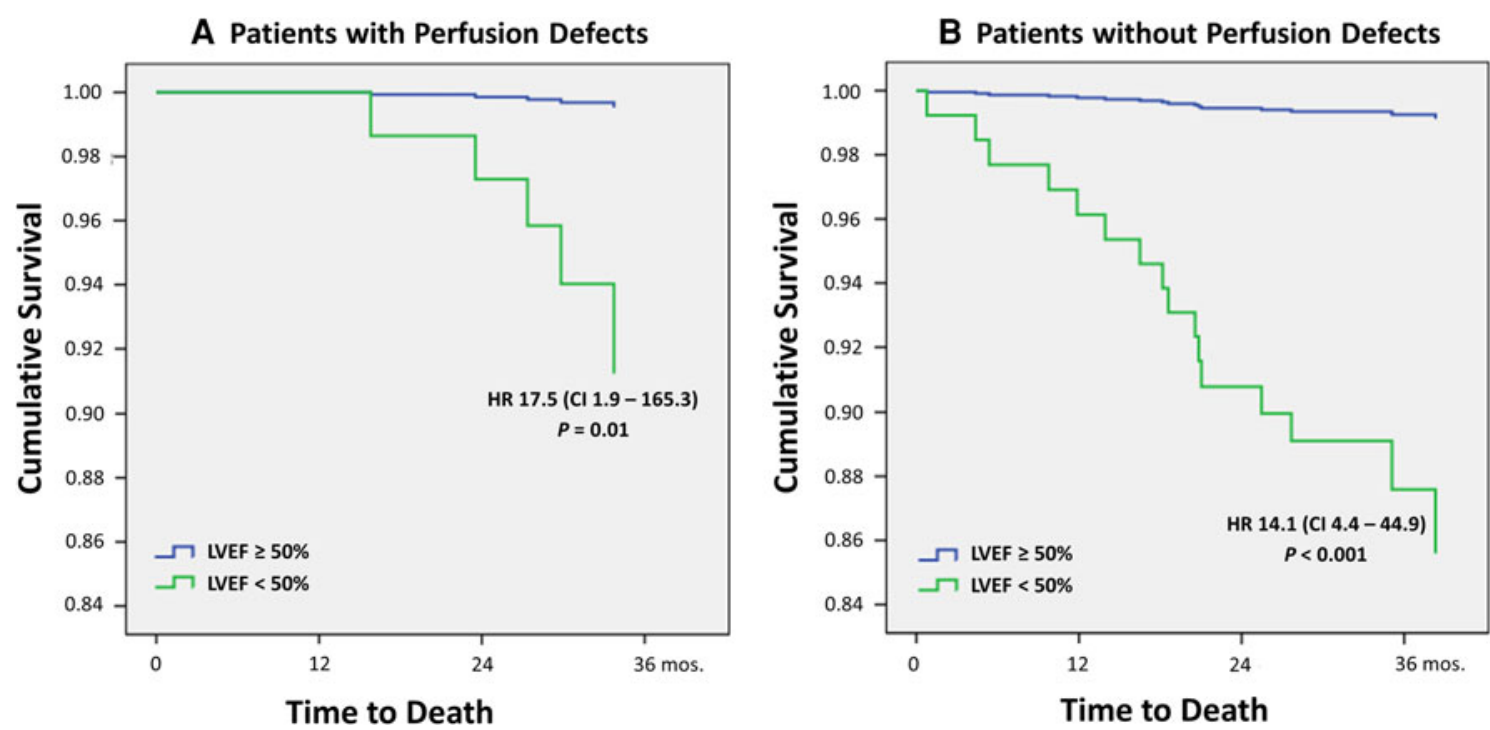

Figure 2. The incremental prognostic value of gated-SPECT LVEF beyond perfusion imaging. Cox proportional hazards survival curves demonstrating the impact of depressed LVEF $(<50 \%)$ on all-cause mortality in patients with and without perfusion abnormalities, adjusted for CAD status and 10-year Framingham CHD risk.
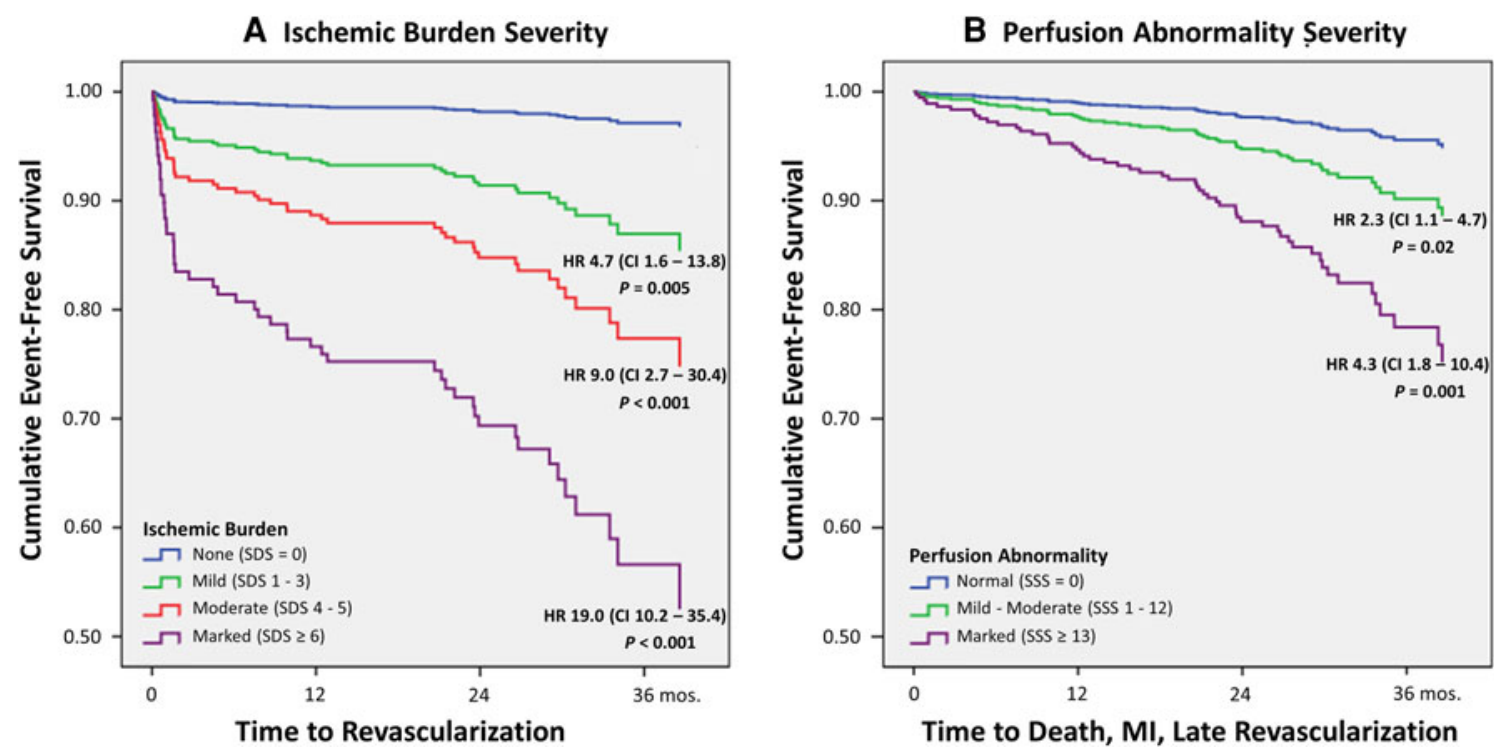

Figure 3. Impact of severity and extent of perfusion abnormalities. Cox proportional hazards event-free survival curves adjusted for CAD status and 10-year Framingham CHD risk. A demonstrates coronary revascularization events are commensurate with ischemic burden (SDS). B demonstrates that all-MACE rates increase commensurately with total perfusion abnormality burden (SSS).

$(P<.001)]$, which translated into higher rates of PCI, CABG, and any revascularization (Table 2). The coronary revascularization rate among patients who underwent coronary angiography after an abnormal perfusion scan was 59\%. Furthermore, the rate of coronary revascularization was commensurate with the burden (size and severity) of stress-induced myocardial ischemia measured by SDS, even after adjusting for Framingham CHD risk and CAD status (Figure 3A). Moreover, abnormal MPI was associated with a higher rate of the composite of death, MI, or late revascularization, even after adjusting for Framingham CHD risk and CAD (Table 2; Figure 1). When MPI was classified into normal (SSS $=0$ ), mild-to-moderately abnormal (SSS 1-12), and 

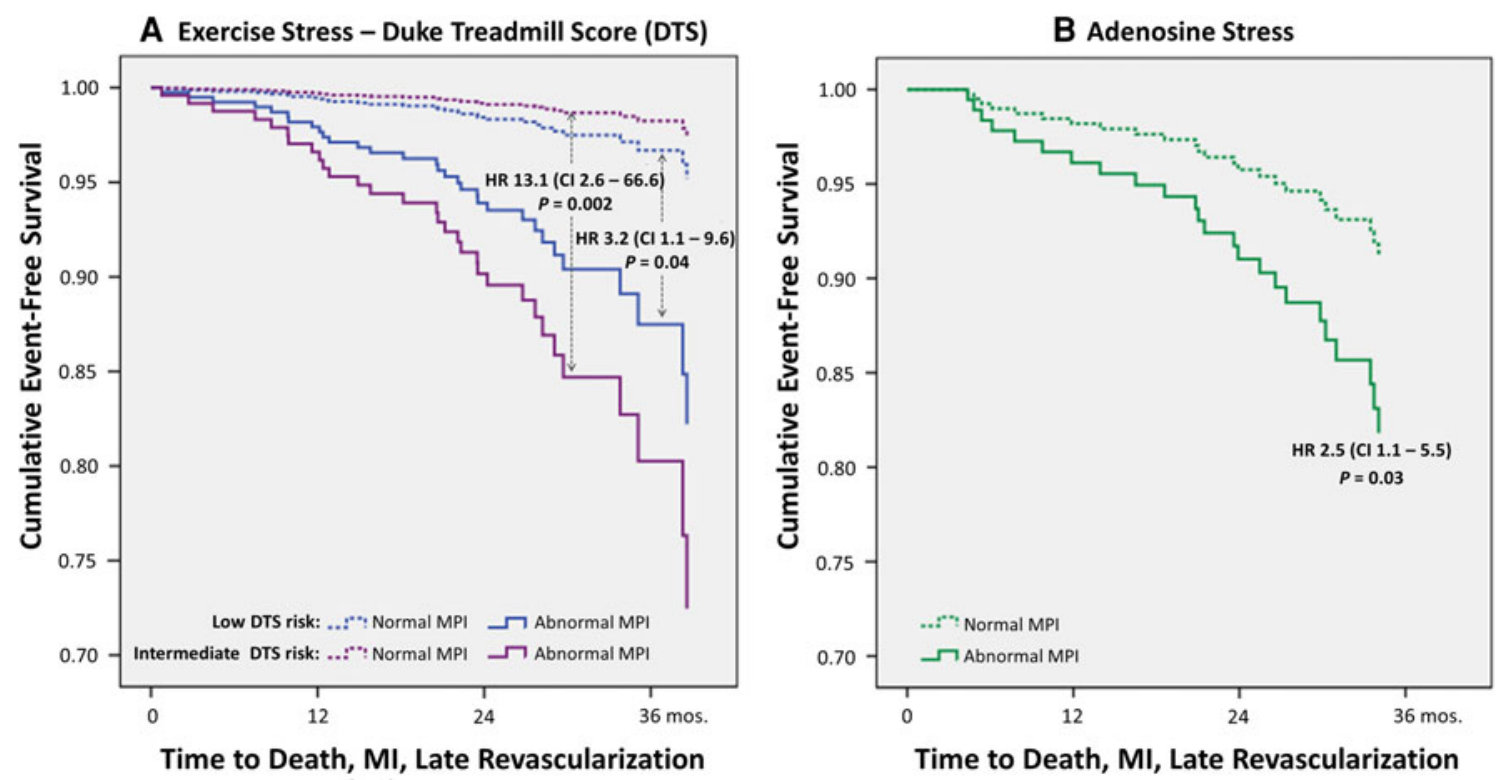

Figure 4. Prognostic value of MPI based on stress modality and Duke treadmill score. Cox proportional hazards survival curves adjusted for CAD status and 10-year Framingham CHD risk.

severely abnormal (SSS $\geq 13$ ), the MACE risk increased concordantly (Figure 3B). Furthermore, TID was an incremental risk predictor for the composite of death, MI, or late revascularization beyond Framingham risk, CAD status, myocardial perfusion, and depressed LVEF status [HR 1.2 per 0.1 point TID increment (CI 1.0-1.3), $P=.001]$.

The inability to perform any level of exercise on the Bruce protocol or with adenosine infusion (AdenoEx) was a very strong predictor of all-cause mortality even after adjusting for CAD status, Framingham CHD risk, perfusion abnormality (SSS), and LVEF; as patients who underwent standard 6-minute adenosine infusion protocol had significantly higher risk of all-cause mortality than those who underwent standard exercise stress [HR 6.3 (CI 2.4-16.5); $P<.001]$ or AdenoEX protocol. However, there was no significant difference in longterm survival between those who underwent a standard exercise stress and those who underwent AdenoEx protocol after adjusting for clinical covariates $(P=.83)$.

MPI was predictive of MACE irrespective of the stress modality used (exercise vs pharmacologic). Among patients who underwent exercise stress, MPI was incrementally predictive of MACE beyond the DTS, particularly among those at intermediate DTS risk (Figure 4A). However, DTS risk category did not provide any incremental prognostic value beyond MPI $(P=.44)$. Furthermore, abnormal MPI was predictive of all-MACE risk among those who underwent adenosine stress protocol (Figure 4B).

In the subgroups of patients whose MPI referral was classified as appropriate or uncertain according to the
2009 AUC ( $\mathrm{N}=736)$, an abnormal MPI was a strong predictor of MACE rates with the exception of MI risk, as shown in Figure 5.

\section{DISCUSSION}

This prospective cohort-study of 1,390 subjects, followed for 27 months, evaluated the prognostic value of SPECT-MPI performed at the PCP's office. The study cohort encompassed a representative sample derived from 10 private primary care practices comprising 20 physicians at various locations within a major metropolitan area. The study demonstrated that stress MPI performed in the setting of the PCP's office using an accredited mobile laboratory maintains an excellent prognostic value similar to what has been previously shown in other care-settings or patient populations. ${ }^{1-5,9,10}$ The study confirms that patients with normal MPI performed in this setting are at a very low annualizedrisk of death $(0.32 \%)$ and death or MI $(0.54 \%)$. Similar to prior reports, ${ }^{7,9}$ we also demonstrated that an abnormal MPI predicted a multifold increase in MACE risk beyond the clinical predictors. In addition, we confirmed that post-stress LVEF and TID ratio provide incremental prognostic value to myocardial perfusion data as it has been established in other settings. ${ }^{5,22}$ Moreover, MACE risk and revascularization rates were commensurate with the total perfusion abnormality burden (SSS) and total ischemic burden (SDS), respectively, reproducing the findings of other investigations. ${ }^{5,9,10}$ These data are the first ever to describe the prognostic value of MPI performed at the PCP's office. 


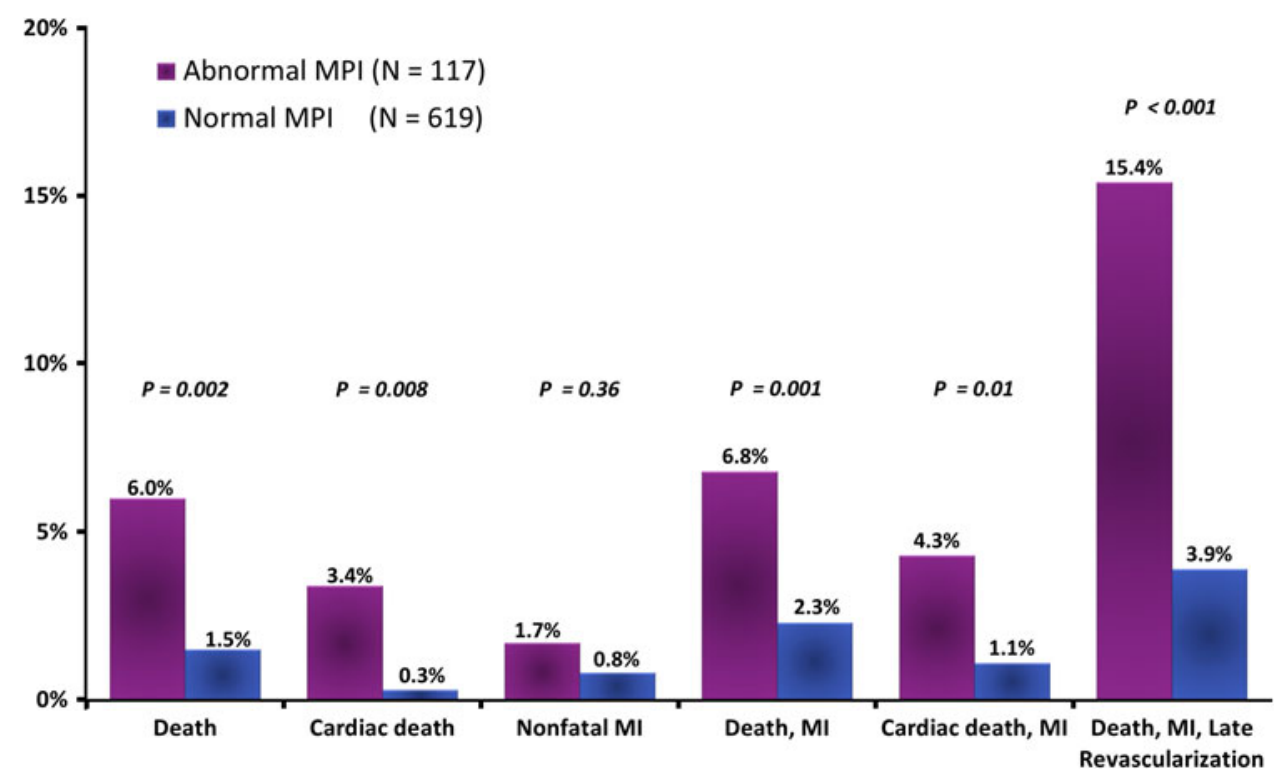

Figure 5. MACE in patients with appropriate or uncertain MPI use.

The study achieved an excellent $99 \%$ follow-up rate among eligible subjects. Those who were excluded or lost to follow-up had similar clinical and imaging characteristics; thus the outcome of this group is unlikely to significantly alter the results of the study.

This investigation defined an abnormal perfusion as SSS $\geq 1$. This is a slight deviation from the conventional definition of SSS $\geq 3$ or 4 used in previous MPI prognosis studies; in which scans with SSS $=1-3$ were considered "near-normal" and were analyzed within the normal group. The reason for this unconventionality is that we attempted to simulate a community practice pattern, in which pursuing additional evaluation, including coronary angiography, is common even for patients with minor abnormalities. In fact, the study confirmed that minimal reversible perfusion abnormality (SDS $=1-3$ ) is associated with the higher likelihood of coronary revascularization than those without any reversible abnormalities (Figure 3A).

It was somewhat surprising that only $17(33 \%)$ of the 51 patients with significant reversible perfusion abnormality (SDS $\geq 6$ ) received coronary revascularization during follow-up (Figure 3A), and only 21 (41\%) subjects underwent coronary angiography. Notably, 18 of the patients who did not receive an angiogram were asymptomatic or had atypical symptoms, and two of them consequently died of non-cardiac cause during follow-up. The low use rate of coronary angiography in patients with marked reversible perfusion abnormality suggests that many of these patients were non-candidates for revascularization in the first place due to age, comorbidity, or lack of symptoms. This sheds light on the possibility of poor patient selection for MPI.

It appears from Figure 2 that patients with decreased LVEF and normal perfusion (presumably non-ischemic cardiomyopathy) have a worse prognosis than those with depressed LVEF and abnormal perfusion (ischemic cardiomyopathy). This is contrary to a large body of published literature. We believe that this is a random, statistically insignificant observation; likely due to the limited number of patients with ischemic cardiomyopathy in the cohort.

The study identified a high non-adherence rate to the 2009 AUC for SPECT-MPI among the participating PCPs, confirming previous reports of higher non-adherence levels among non-cardiologists. ${ }^{23}$ However, the observed $47 \%$ rate seems to be decisively higher than the $19.5 \%$ non-adherence rate among non-cardiologists reported by Hendel et al and other published AUC reports. ${ }^{23-27}$ High non-adherence level in our study may be, in part, related to suboptimal documentation of test rational by the referring physicians, lack of awareness of the AUC at the time of testing (2007-2010), and possible self-referral biases. On the other hand, due to publication bias, the published AUC adherence reports represent "best-case scenarios" and certainly are not reflective of adherence practices in other institutions or at the community level. Even the prospective broad-base study by Hendel et al may have preselected practices and physicians with higher awareness of AUC and better adherence habits than is commonplace. In addition, physicians' mindfulness that they were being "scored" 
for their AUC adherence in Hendel's study could have improved their adherence level. Nonetheless, the high inappropriate use rate in this study and the great disparity in adherence rates between physicians are rather surprising. Therefore, this study further emphasizes the role of the AUC as an important quality measure in the laboratory accreditation process, which has already been implemented by the Intersocietal

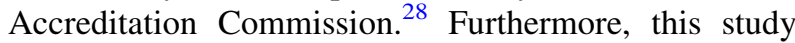
emphasizes the need for national organizations of primary care to educate their members about the AUC.

The impact of the adherence to the AUC on the prognostic value of MPI is of utmost importance. In this investigation, MPI maintained a robust prognostic value in the subgroup of patients with appropriate / uncertain use (Figure 5). In these analyses, we did not adjust for Framingham's risk or CAD status since these variables were already accounted for in the determination of AUC classification. The subgroup of patients with inappropriate use was underpowered to undergo such analyses.

The cohort's subjects were relatively young and healthy with low CHD risk and CAD prevalence. It is remarkable that $79 \%$ of the patients underwent exercise stress, among which $78 \%$ fell in a low DTS risk category. This is certainly a deviation from reports of other cohorts. For example, the NUC study by Thomas et $\mathrm{al}^{5}$ reported a $63 \%$ utilization rate of exercise stress among subjects who underwent an out-patient stressMPI, while a study by Hachamovitch et al $^{7}$ described only $40 \%$ of its population to be at low DTS risk. These differences highlight the fact that our study population was healthier than those of previous MPI prognostic studies. Undoubtedly, the low risk of the tested population is directly related to the high inappropriate use rate.

The investigation was designed as an outcome study, and we did not intend to evaluate the diagnostic accuracy of SPECT-MPI in this setting. However, the demonstrated prognostic performance and high revascularization yield following an abnormal MPI suggest a favorable diagnostic accuracy of SPECT-MPI in this setting. Furthermore, early coronary revascularizations ( $\leq 60$ days post-MPI) were not considered MACE since they are often prompted by the MPI findings. As we have learned from the COURAGE trial, it is unlikely for early revascularizations to significantly impact the rate of death or MI in patients with stable disease, but they may have decreased the rate of late ( $>60$ days) revascularization procedures. ${ }^{29}$ Therefore, not accounting for early revascularizations as MACE may have lowered the rate of the composite endpoint of death, MI, or late revascularization; biasing this analysis to the null and, thus, strengthening the study's conclusions.
This study is primarily limited by the low rate of hard-events, particularly nonfatal MI and cardiac death, which can be explained by the relative health and youth of the study cohort. This limitation was overcome by an adequate rate of the composite endpoint of death, MI, and late revascularization. Furthermore, the study came slightly short of the targeted enrollment goal of 1,500 subjects, which was compensated for by longer followup than the originally planned 24 months. Finally, having all scans interpreted by a single reader is another limitation.

\section{CONCLUSION}

High-quality and expertly interpreted SPECT-MPI performed in the office of a community-based PCP carries a similar prognostic value to that demonstrated in tertiary or large-volume practice settings. This study suggests that the adherence rate to the AUC for MPI at the PCP's office is low.

\section{Acknowledgments}

The authors are deeply grateful to all the participating physicians and their office staff whose collaboration made this study possible.

\section{Conflicts of interest}

Rami Doukky is the medical director of Mobile Nuclear Cardiology Laboratory. Robert Hendel serves on the Advisory Board for Astellas Pharma US.

\section{References}

1. Doukky R, Hendel RC. Practical guide to risk stratification using myocardial perfusion imaging. In: Heller GV, Hendel R, editors. Nuclear cardiology: Practical applications. New York: McGrawHill; 2004. p. 259-74.

2. Iskander S, Iskandrian AE. Risk assessment using single-photon emission computed tomographic technetium-99m sestamibi imaging. J Am Coll Cardiol 1998;32:57-62.

3. Shaw LJ, Berman DS, Hendel RC, Alazraki N, Krawczynska E, Borges-Neto $\mathrm{S}$, et al. Cardiovascular disease risk stratification with stress single-photon emission computed tomography technetium$99 \mathrm{~m}$ tetrofosmin imaging in patients with the metabolic syndrome and diabetes mellitus. Am J Cardiol. 2006;97(10):1538-44.

4. Shaw LJ, Hendel R, Borges-Neto S, Lauer MS, Alazraki N, Burnette J, et al. Prognostic value of normal exercise and adenosine (99m)Tc-tetrofosmin SPECT imaging: Results from the multicenter registry of 4,728 patients. J Nucl Med. 2003;44:134-9.

5. Thomas GS, Miyamoto MI, Morello AP 3rd, Majmundar H, Thomas JJ, Sampson CH, et al. Technetium 99m sestamibi myocardial perfusion imaging predicts clinical outcome in the community outpatient setting. The Nuclear Utility in the Community (NUC) Study. J Am Coll Cardiol. 2004;43:213-23. 
6. Boiten HJ, van der Sijde JN, Ruitinga PR, Valkema R, Geleijnse ML, Sijbrands EJ, et al. Long-term prognostic value of exercise technetium-99m tetrofosmin myocardial perfusion single-photon emission computed tomography. J Nucl Cardiol. 2012;19:907-13.

7. Hachamovitch R, Berman DS, Kiat H, Cohen I, Cabico JA, Friedman $\mathrm{J}$, et al. Exercise myocardial perfusion SPECT in patients without known coronary artery disease: Incremental prognostic value and use in risk stratification. Circulation 1996;93:905-14.

8. Iskandrian AS, Chae SC, Heo J, Stanberry CD, Wasserleben V, Cave V. Independent and incremental prognostic value of exercise single-photon emission computed tomographic (SPECT) thallium imaging in coronary artery disease. J Am Coll Cardiol 1993;22:665-70.

9. Vanzetto G, Ormezzano O, Fagret D, Comet M, Denis B, Machecourt J. Long-term additive prognostic value of thallium201 myocardial perfusion imaging over clinical and exercise stress test in low to intermediate risk patients: Study in 1137 patients with 6-year follow-up. Circulation 1999;100:1521-7.

10. Hachamovitch R, Berman DS, Shaw LJ, Kiat H, Cohen I, Cabico JA, et al. Incremental prognostic value of myocardial perfusion single photon emission computed tomography for the prediction of cardiac death: Differential stratification for risk of cardiac death and myocardial infarction. Circulation 1998;97:535-43.

11. Chaptini N, Dontaraju VS, Concepcion F, Muradi HA, Alyousef T. Del Pilar Aristizabal Canaval M, et al. Ambulatory cardiac singlephoton emission computed tomography at the primary care physician's office: A descriptive study. J Ambul Care Manage. 2010;33:328-35

12. Sox HC Jr, Hickam DH, Marton KI, Moses L, Skeff KM, Sox CH, et al. Using the patient's history to estimate the probability of coronary artery disease: A comparison of primary care and referral practices. Am J Med 1990;89:7-14.

13. Simon D, Boring JR. Sensitivity, specificity and positive predictive value. In: Walker HK, Hall WD, Hurst W, editors. Clinical methods. 3rd ed. Boston, MA: Butterworths; 1990. p. 49-54.

14. Wilson PW, D'Agostino RB, Levy D, Belanger AM, Silbershatz H, Kannel WB. Prediction of coronary heart disease using risk factor categories. Circulation 1998;97:1837-47.

15. Diamond GA, Forrester JS. Analysis of probability as an aid in the clinical diagnosis of coronary-artery disease. N Engl J Med 1979;300:1350-8.

16. Hendel RC, Berman DS, Di Carli MF, Heidenreich PA, Henkin RE, Pellikka PA, et al. ACCF/ASNC/ACR/AHA/ASE/SCCT/ SCMR/SNM 2009 Appropriate Use Criteria for Cardiac Radionuclide Imaging: A Report of the American College of Cardiology Foundation Appropriate Use Criteria Task Force, the American Society of Nuclear Cardiology, the American College of Radiology, the American Heart Association, the American Society of Echocardiography, the Society of Cardiovascular Computed Tomography, the Society for Cardiovascular Magnetic Resonance, and the Society of Nuclear Medicine. Endorsed by the American College of Emergency Physicians. J Am Coll Cardiol. 2009;53: 2201-29.
17. Henzlova MJ, Cerqueira MD, Mahmarian JJ, Yao SS. Stress protocols and tracers. J Nucl Cardiol. 2006;13:e80-90.

18. Doukky R. Pharmacologic stress testing in myocardial perfusion imaging: Technical applications. In: Mann A, Heller GV, Hendel RC, editors. Nuclear cardiology: Technical applications. New York: McGraw-Hill; 2007. p. 107-24.

19. Elliott MD, Holly TA, Leonard SM, Hendel RC. Impact of an abbreviated adenosine protocol incorporating adjunctive treadmill exercise on adverse effects and image quality in patients undergoing stress myocardial perfusion imaging. J Nucl Cardiol. 2000;7(6):584-9.

20. Mark DB, Shaw L, Harrell FE Jr, Hlatky MA, Lee KL, Bengtson JR, et al. Prognostic value of a treadmill exercise score in outpatients with suspected coronary artery disease. N Engl J Med 1991;325:849-53

21. Cerqueira MD, Weissman NJ, Dilsizian V, Jacobs AK, Kaul S, Laskey WK, et al. Standardized myocardial segmentation and nomenclature for tomographic imaging of the heart: A statement for healthcare professionals from the Cardiac Imaging Committee of the Council on Clinical Cardiology of the American Heart Association. Circulation. 2002;105:539-42.

22. Sharir T, Germano G, Kavanagh PB, Lai S, Cohen I, Lewin HC, et al. Incremental prognostic value of post-stress left ventricular ejection fraction and volume by gated myocardial perfusion single photon emission computed tomography. Circulation 1999;100:1035-42.

23. Hendel RC, Cerqueira M, Douglas PS, Caruth KC, Allen JM, Jensen NC, et al. A multicenter assessment of the use of singlephoton emission computed tomography myocardial perfusion imaging with appropriateness criteria. J Am Coll Cardiol 2010;55: 156-62.

24. Koh AS, Flores JL, Keng FY, Tan RS, Chua TS. Evaluation of the American College of Cardiology Foundation/American Society of Nuclear Cardiology appropriateness criteria for SPECT myocardial perfusion imaging in an Asian tertiary cardiac center. J Nucl Cardiol 2011;18:324-30.

25. Carryer DJ, Askew JW, Hodge D, Miller TD, Gibbons RJ. The impact of ordering provider specialty on appropriateness classification. J Nucl Cardiol 2012;19:285-90.

26. Carryer DJ, Hodge DO, Miller TD, Askew JW, Gibbons RJ. Application of appropriateness criteria to stress single photon emission computed tomography sestamibi studies: A comparison of the 2009 revised appropriateness criteria to the 2005 original criteria. Am Heart J 2010;160:244-9.

27. Gupta A, Tsiaras SV, Dunsiger SI, Tilkemeier PL. Gender disparity and the appropriateness of myocardial perfusion imaging. J Nucl Cardiol 2011;18:588-94.

28. Intersocietal Accreditation Commission. The IAC Standards and Guidelines for Nuclear/PET Accreditation. 08/2012; 42-4. Available from http://www.intersocietal.org/nuclear/standards/ IACNuclearPETStandards2012.pdf. Accessed 17 Dec 2012

29. Boden WE, O'Rourke RA, Teo KK, Hartigan PM, Maron DJ, Kostuk WJ, et al. Optimal medical therapy with or without PCI for stable coronary disease. N Engl J Med 2007;356:1503-16. 